\title{
Role of Nanoscale Precipitates on the Enhanced Magnetostriction of Heat-Treated Galfenol $\left(\mathrm{Fe}_{1-x} \mathbf{G a}_{x}\right)$ Alloys
}

\author{
H. Cao, ${ }^{1}$ P. M. Gehring, ${ }^{2}$ C. P. Devreugd, ${ }^{1}$ J. A. Rodriguez-Rivera, ${ }^{3}$ J. Li, ${ }^{1}$ and D. Viehland ${ }^{1}$ \\ ${ }^{1}$ Department of Materials Science and Engineering, Virginia Tech, Blacksburg, Virginia 24061, USA \\ ${ }^{2}$ NIST Center for Neutron Research, NIST, Gaithersburg, Maryland 20899-6100, USA \\ ${ }^{3}$ Department of Materials Science and Engineering, University of Maryland, College Park, Maryland 20742, USA
}

(Received 10 May 2008; revised manuscript received 22 December 2008; published 25 March 2009)

\begin{abstract}
We report neutron diffuse scattering measurements on highly magnetostrictive $\mathrm{Fe}_{1-x} \mathrm{Ga}_{x}$ alloys $(0.14<$ $x<0.20$ ) with different thermal treatments. This diffuse scattering scales with magnetostriction and exhibits asymmetric peaks at the (100) and (300) reciprocal lattice positions that are consistent with the coexistence of short-range ordered, coherent nanometer-scale precipitates embedded in a long-range ordered, body-centered cubic matrix. A large peak splitting is observed at (300) for $x=0.19$, which indicates that the nanoprecipitates are not cubic and have a large elastic strain. This implies a structural origin for the enhanced magnetostriction.
\end{abstract}

DOI: 10.1103/PhysRevLett.102.127201

Mechanically strong and malleable Galfenol alloys $\left(\mathrm{Fe}_{1-x} \mathrm{Ga}_{x}\right)$ exhibit enhanced and extremely large magnetostriction coefficients along [100] of up to $3 \lambda_{100} / 2=$ $400 \mathrm{ppm}$ at low saturation fields [1,2]. The addition of Ga into the body-centered cubic (bcc) $\alpha$-Fe phase is known to produce a diversity of crystal structures including chemically disordered bcc $A 2(\mathrm{Fe})$, ordered bcc $\mathrm{DO}_{3}$ $\left(\mathrm{Fe}_{3} \mathrm{Ga}\right)$, ordered bcc $B_{2}(\mathrm{FeGa})$, and face-centered cubic $L 1_{2}\left(\mathrm{Fe}_{3} \mathrm{Ga}\right)$ phases $[3,4]$. Previous studies of $\mathrm{Fe}_{1-x} \mathrm{Ga}_{x}$ alloys cooled (postgrowth) at $10^{\circ} \mathrm{C} / \mathrm{min}$ have shown the presence of two anomalous peaks in the magnetostriction. The first peak at $x \approx 0.19$ has been attributed to an increase in the magnetoelastic coupling, resulting from the formation of short-range ordered (SRO) Ga pairs along the [100] axis of the $A 2$ structure [5], and the second at $x \approx 0.27$ to a softening of the shear modulus $c^{\prime}=\left(c_{11}-c_{12}\right) / 2[6,7]$. Of special note is the fact that both $3 \lambda_{100} / 2$ and the presence of a two-phase region $\left(A 2+\mathrm{DO}_{3}\right)$ depend sensitively on thermal history; this suggests that the enhanced magnetostriction is due to an underlying heterogeneity rather than a conventional homogeneous ferromagnetic phase.

A structurally heterogeneous model [8] has been proposed to explain the enhanced magnetostriction and elastic softening for such $\mathrm{Fe}_{1-x} \mathrm{Ga}_{x}$ alloys. This model assumes that the heat treatment produces a structurally and chemically heterogeneous state consisting of coarseningresistant, nanometer-scale $\mathrm{DO}_{3}$ precipitates within an $A 2$ matrix. Theoretically, heterogeneity has been predicted to result from the following sequence of transformations: (i) bcc $\rightarrow \mathrm{bcc}^{\prime}+\mathrm{DO}_{3}$ decomposition, followed by (ii) a diffusionless Bain strain that transforms the $\mathrm{DO}_{3}$ nanoprecipitates into a face-centered tetragonal (fct) structure. Recently, nanometer-scale $(<2 \mathrm{~nm}) \mathrm{DO}_{3}$ precipitates [9] have been identified in an $A 2$ matrix of $\mathrm{Fe}_{0.81} \mathrm{Ga}_{0.19}$ using high-resolution transmission electron microscopy (HRTEM), consistent with the theoretical prediction of step (i) in the transformational sequence. However, all
PACS numbers: 75.80.+q, 61.05.fg, 64.70.Nd, 75.50.Bb

prior structural investigations have shown that both the $\mathrm{DO}_{3}$ and $A 2$ phases are cubic. There have been no reports of lower-symmetry phases, as predicted by step (ii) in the transformational sequence. This is a key point because without the lower-symmetry $\mathrm{DO}_{3}$ nanodispersion with a confined elastic distortion, the structural heterogeneity cannot contribute to the enhanced magnetostriction.

Here we report neutron diffuse scattering measurements on $\mathrm{Fe}_{1-x} \mathrm{Ga}_{x}$ alloys $(x=0.10,0.15,0.19,0.22$, and 0.25$)$ as a function of thermal treatment. We observed strong diffuse scattering within the $A 2+\mathrm{DO}_{3}$ two-phase region $(0.14<x<0.22)$ for $x=0.19$ cooled (postgrowth) at $10^{\circ} \mathrm{C} /$ min that is absent when cooled at $2{ }^{\circ} \mathrm{C} / \mathrm{min}$; this composition exhibits the strongest diffuse scattering and the highest value of $3 \lambda_{100} / 2$, thus confirming that the enhanced magnetostriction is directly related to the presence of an underlying structural heterogeneity. Furthermore, the diffuse scattering intensity exhibits asymmetric peaks near (100) and (300), the latter of which is split along [100]. These findings indicate that the coherent nanoprecipitates that form within the $A 2$ matrix are not cubic but rather of tetragonal (or lower) symmetry.

Single crystal $\mathrm{Fe}_{1-x} \mathrm{Ga}_{x}$ alloys cut into cubes $(10 \mathrm{~mm} \times$ $10 \mathrm{~mm} \times 10 \mathrm{~mm}$ ) with $\langle 100\rangle$-oriented faces were grown at Ames Lab using a Bridgman method previously described [10]. Three thermal treatments were applied to these crystals: We shall refer to them by the designations "asgrown" (cooled at $10^{\circ} \mathrm{C} / \mathrm{min}$ during crystal growth), "furnace-cooled" (cooled at $10^{\circ} \mathrm{C} / \mathrm{min}$, postgrowth), and "slow-cooled" (cooled at $2{ }^{\circ} \mathrm{C} / \mathrm{min}$, postgrowth). Crystals were first heated to $1100^{\circ} \mathrm{C}$ at $10^{\circ} \mathrm{C} / \mathrm{min}$ and then annealed at $1100^{\circ} \mathrm{C}$ for 3 hours under vacuum before cooling. All neutron diffuse scattering experiments were performed on the BT7 and BT9 triple-axis spectrometers located at the NIST Center for Neutron Research. The scattering data were taken using fixed incident neutron energies of either $E_{i}=14.7$ or $30.5 \mathrm{meV}$. The (002) Bragg 
reflection of highly oriented pyrolytic graphite (HOPG) crystals was used to define the incident and scattered neutron energies, and HOPG filters were placed before and after the sample to remove unwanted contamination from neutrons with wavelengths $\lambda / 2$ and $\lambda / 3$. The horizontal angular beam collimations on BT7 were open-50'-S-40'$180^{\prime}$ (" $S$ " refers to the sample), whereas the collimations on BT9 were $40^{\prime}-47^{\prime}-S-40^{\prime}-\left(40^{\prime} / 80^{\prime}\right)$. The room temperature (cubic) lattice parameter of $\mathrm{Fe}_{0.81} \mathrm{Ga}_{0.19}$ is $a_{c}=$ $2.900 \AA$; thus, one reciprocal lattice unit $(1 \mathrm{rlu})$ equals $a_{c}{ }^{*}=2 \pi / a_{c}=2.167 \AA^{-1}$. All data presented are plotted with respect to this cubic lattice parameter in terms of rlu.

Figure 1 shows diffuse scattering intensity contours (log scale) measured in the [100]-[001] plane near the reciprocal lattice vector $\mathbf{Q}=(100)$ at room temperature for (a) asgrown $\mathrm{Fe}_{0.81} \mathrm{Ga}_{0.19}$, (b) slow-cooled $\mathrm{Fe}_{0.81} \mathrm{Ga}_{0.19}$, and (c) as-grown $\mathrm{Fe}_{0.75} \mathrm{Ga}_{0.25}$. As-grown $\mathrm{Fe}_{0.81} \mathrm{Ga}_{0.19}$ exhibits substantial diffuse scattering intensity that is broad in both

$$
\mathrm{Fe}_{1-\mathrm{x}} \mathrm{Ga}_{\mathrm{x}},(100), 300 \mathrm{~K}, \mathrm{E}_{\mathrm{i}}=14.7 \mathrm{meV}
$$

BT-7,open-50'-40'-180', collecting time $=3 \mathrm{~min} / \mathrm{pt}$

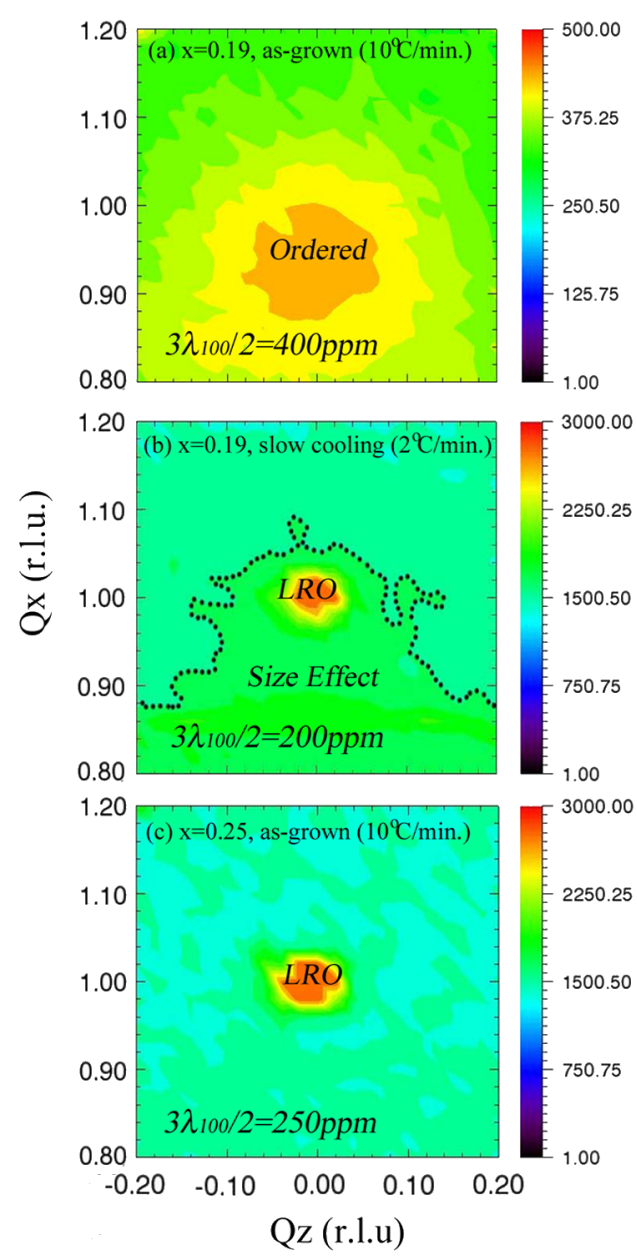

FIG. 1 (color online). Diffuse scattering intensity contours (log scale) measured in air on BT7 at room temperature for (a) asgrown $\mathrm{Fe}_{0.81} \mathrm{Ga}_{0.19}$, (b) slow-cooled $\mathrm{Fe}_{0.81} \mathrm{Ga}_{0.19}$, and (c) asgrown $\mathrm{Fe}_{0.75} \mathrm{Ga}_{0.25}$.
$Q_{x}$ and $Q_{z}$ and thus consistent with the presence of ordered coherent nanoprecipitates. In addition, the diffuse peak is not centered on (100) but rather appears at $Q_{x}=0.965$. By contrast, slow-cooled $\mathrm{Fe}_{0.81} \mathrm{Ga}_{0.19}$ exhibits a sharply defined Bragg peak, centered on (100), while a weak envelope of diffuse scattering is seen that extends to lower $Q$ [see Fig. 1(b)]. For as-grown $\mathrm{Fe}_{0.75} \mathrm{Ga}_{0.25}$, the diffuse scattering intensity contours [see Fig. 1(c)] are similar to those for $x=0.19$ in the slow-cooled condition. Given that a (100) superlattice reflection is forbidden in the $A 2$ and $L 1_{2}$ phases, we can infer that (i) either the $\mathrm{DO}_{3}$ or the $B 2$ phase is the structural origin of the (100) Bragg peak, (ii) ordered $\mathrm{DO}_{3}$ (or $B 2$ ) coherent nanoprecipitates decompose out during the as-grown and/or furnace-cooled conditions for $0.14<x<0.20$, and (iii) a long-range ordered (LRO) $\mathrm{DO}_{3}$ phase decomposes out of the $A 2$ matrix on slow cooling for $x>0.19$, as previously reported in Ref. [11].

Next, we investigated the effect of Ga content $x$ on the decomposition of coherent nanoprecipitates from the $A 2$ matrix. Diffuse scattering intensities were measured in the [100]-[001] plane along [100] near (100) for $x=0.10$, 0.15 , and 0.19 in the as-grown condition, as shown in Fig. 2(a). Our main result concerns the strong dependence of the diffuse scattering intensity on Ga content. For $x=$ 0.10 , weak and featureless diffuse scattering is observed
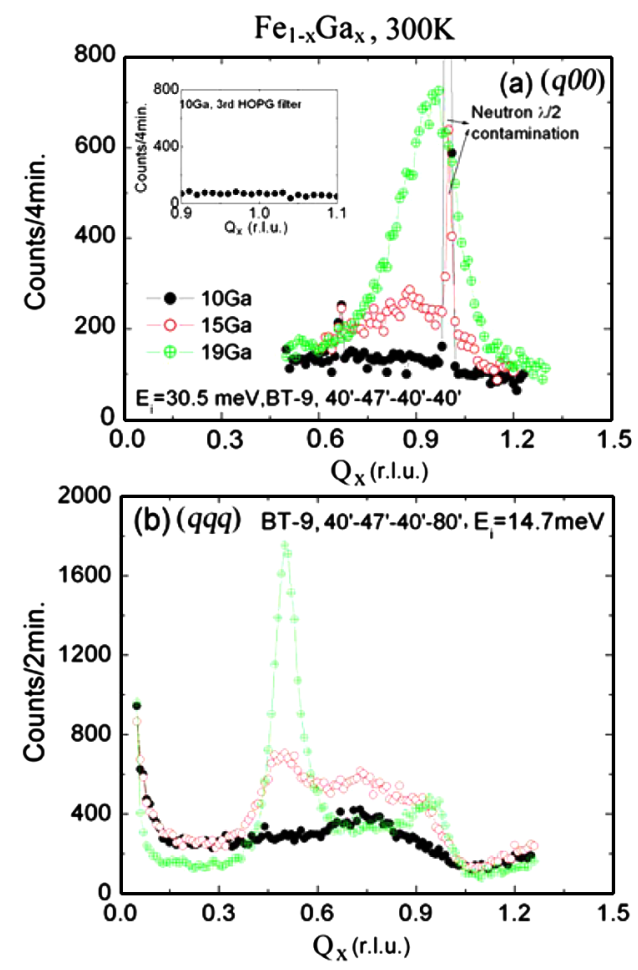

FIG. 2 (color online). Diffuse scattering profiles, measured in air on BT9 near (a) (100) along [100] and (b) $(1 / 21 / 21 / 2)$ along [111] at room temperature, for as-grown $\mathrm{Fe}_{1-x} \mathrm{Ga}_{x}(x=$ $0.10,0.15$, and 0.19). The sharp peaks centered at $Q_{x}=1$ and $Q_{x}=0.67$ result from higher order neutron contaminations; the addition of a third HOPG filter eliminated these peaks as shown for $x=0.10$ at $Q_{x}=1$ in the inset. 
where the scattering profile is almost flat for $0.5<Q_{x}<$ 1.3. For $x=0.15$, a weak and broad peak is seen, indicating the formation of ordered coherent nanoprecipitates. The intensity of this broad peak increases markedly for $x=0.19$, which is consistent with the intensity contours of Fig. 1(a). Identical scans for $x=0.22$ (not shown) reveal sharp Bragg peaks centered exactly on (100), as can be seen in the contour plot for as-grown $\mathrm{Fe}_{0.75} \mathrm{Ga}_{0.25}$ shown in Fig. 1(c). Several other features for $\mathrm{Fe}_{0.81} \mathrm{Ga}_{0.19}$ in the asgrown condition shown in Figs. 1(a) and 2(a) are noteworthy. First, the diffuse scattering intensity contours are nearly isotropic, which indicates that the short-range structural correlations along [100] $\left(Q_{x}\right)$ and [001] $\left(Q_{z}\right)$ are roughly of equal extent (i.e., equal-axial nanoprecipitates). This implies that the approximate real-space shape of the coherent nanoprecipitates is spherical, which would be consistent with the morphology measured by electron microscopy [3]. Second, the peak in the diffuse scattering, while centered at $Q_{z}=0$, is slightly offset to $Q_{x}<1$. This peak shifts slowly towards $Q_{x}=1$ with increasing $x$ for $0.15<x<0.19$, becoming centered at $Q_{x}=1$ for $x=$ 0.22 . Finally, for $x=0.15$ and 0.19 , a notable peak asymmetry along the [100] direction can be seen in Fig. 2(a). Recent neutron diffraction studies of $\mathrm{Fe}_{1-x} \mathrm{Ga}_{x}$ alloy ribbons have also revealed a $\mathrm{DO}_{3}$ phase with a similar peak asymmetry and a possible shift of the superlattice peak position away from $Q_{x}=1$ [12].

Diffuse scattering intensities were also measured in the [100]-[011] reciprocal space plane along [111] for $x=$ $0.10,0.15$, and 0.19 in the as-grown condition and are shown in Fig. 2(b). Upon increasing the Ga content from $x=0.10$ to $x=0.19$, a broad peak appears at $(1 / 21 / 21 / 2)$ that increases more than eightfold in intensity while narrowing by nearly the same amount. These data (i) eliminate the possibility of $B 2$ ordering as the origin of the (100) Bragg peak, as the $(1 / 21 / 21 / 2)$ superlattice reflection occurs only for the $\mathrm{DO}_{3}$ structure, and (ii) demonstrate that the size of coherent $\mathrm{DO}_{3}$ nanoprecipitates increases dramatically on approaching the $A 2-\mathrm{DO}_{3}$ boundary. Further, the linewidth determined from the $(1 / 21 / 21 / 2)$ diffuse peak for $x=19$ corresponds to a correlation length of $1.1 \mathrm{~nm}$, which is consistent with results from the HRTEM study [9].

Two interesting differences between the (100) and $(1 / 21 / 21 / 2)$ diffuse peaks are apparent for $\mathrm{Fe}_{0.81} \mathrm{Ga}_{0.19}$. First, the $(1 / 21 / 21 / 2)$ peak is symmetric and well described by a Lorentzian function of $Q$, whereas the (100) peak is notably asymmetric; second, the $(1 / 21 / 21 / 2)$ peak is commensurate with the fundamental periodicity of $A 2$, whereas the (100) peak is not. Bragg peak asymmetry in binary alloys with short-range ordering has previously been attributed to size-effect diffuse scattering in single-phase homogeneous solutions $[13,14]$, which adds a sinusoidal contribution to the diffraction intensity. In addition, the position of a diffuse peak can be shifted to lower $Q$ in a binary alloy if the atom with the larger atomic size $\varepsilon$ has a greater neutron scattering length $b$ (i.e., if $\varepsilon_{a-a}>\varepsilon_{b-b}$ and $\left.b_{a}>b_{b}\right)$ [13]. That the $(1 / 21 / 21 / 2)$ diffuse peak intensity is symmetric and commensurate indicates that the size-effect diffuse scattering contribution in this zone is small. The ordering within the coherent nanoprecipitates can therefore be considered to be quite uniform, consisting of the stacking of a finite number of periodically alternating (111) atomic $A A A B$ or $\mathrm{FeFeFeGa}$ layers [8]. However, the (100) diffuse peak is notably asymmetric and shifted to lower $Q$. Superlattice reflections in this zone are generated by diffraction from a finite number of periodically alternating (100) atomic $A B$ or $\mathrm{Fe}\left(\mathrm{Fe}_{0.5} \mathrm{Ga}_{0.5}\right)$ layers. We could attribute the (100) asymmetry and the peak shift to static-displacement modulations in the $B$ layers or, alternatively, to interstitial planar defects that alter the stacking sequence, such as $\mathrm{Fe}\left(\mathrm{Fe}_{0.5} \mathrm{Ga}_{0.5}\right) \mathrm{Fe}$.

If the asymmetry of the (100) diffuse peak is due only to a size effect in a homogenous $\mathrm{Fe}_{1-x} \mathrm{Ga}_{x}$ solution, then the diffuse scattering peaks located at superlattice positions of different order should have asymmetric peak widths that are a multiple of the order of the reflection. We show in Figs. 3(a) and 3(b) the peak asymmetry observed in scans measured along [100] at (100) $\left(E_{i}=14.7 \mathrm{meV}\right)$ and (300) $\left(E_{i}=30.5 \mathrm{meV}\right)$ for $x=0.19$. A mechanical limit on the maximum scattering angle constrained measurements to
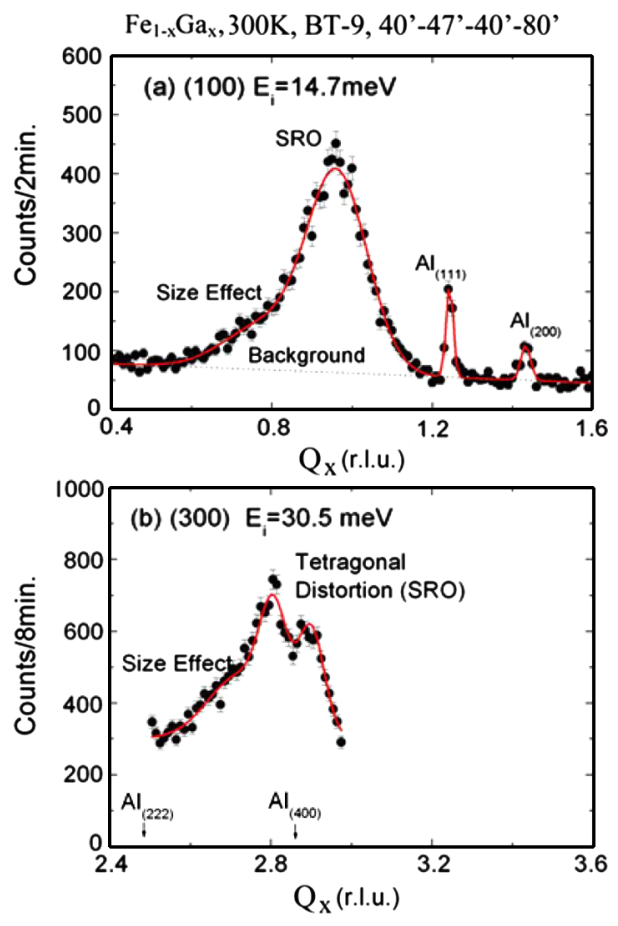

FIG. 3 (color online). Diffuse scattering profiles for furnacecooled $\mathrm{Fe}_{0.81} \mathrm{Ga}_{0.19}$ at $300 \mathrm{~K}$ measured on BT9 near (a) (100) and (b) (300). The black dotted line in (a) represents the background. The solid lines through the data points in (a) and (b) represent fits using multi-Gaussian functions. The sharp peaks near $q=1.2$ and 1.45 in (a) are the (111) and (200) reflections from the $\mathrm{Al}$ wire used to mount the sample: The positions of the $\mathrm{Al}$ (222) and (400) reflections are indicated in the (300) profile in (b). 
the low- $Q$ side of (300). A comparison of the (100) and (300) diffuse peaks reveals (i) broad shoulders to the left side of the principle diffuse scattering peaks, centered near $Q_{x}=0.65$ and $Q_{x}=2.7$, respectively (i.e., the shifts are nearly the same) and (ii) no significant change in the widths of the asymmetric peaks. These results indicate that peak asymmetries are independent of the order of the zone and thus that size effects in a homogenous $\mathrm{Fe}_{1-x} \mathrm{Ga}_{x}$ solution alone cannot explain the observations. Rather, the solution should be viewed as inhomogeneous: Diffraction occurs from coherent nanoprecipitates with a $\mathrm{DO}_{3}$-like structure, which may have some $B 2$ component, imbedded in a $A 2$ matrix.

The data in Fig. 3(b) also reveal a splitting of the (300) diffuse peak into two peaks centered near $Q_{x}=2.80$ and $Q_{x}=2.90$. This unambiguously demonstrates the presence of an ordered distortion from cubic symmetry for $x=$ 0.19 . The absence of a well-defined peak splitting near (100) is due to the broad peak widths, which make it impossible to resolve a peak centered at $Q_{x}=2.80 / 3=$ 0.933 from one centered at $Q_{x}=2.90 / 3=0.967$. We note that (i) the (100) peak is centered at $Q_{x} \sim 0.95$, which is nearly the average of the positions corresponding to those of the two (300) peaks, and (ii) the (100) peak width is actually broader than that of (300), consistent with the presence of two unresolved peaks. Such a splitting suggests the nanoprecipitates are tetragonally distorted. The fact that no splitting or asymmetry is seen at $(1 / 21 / 21 / 2)$ is consistent with this picture because a tetragonal distortion cannot split reflections of the form $(h h h)$. We can estimate the tetragonality from the (300) splitting to be $c / a \approx 1.04$, which is a relatively large local distortion within an average cubic system. This finding supports the predictions of step (ii) in the transformation sequence of the structurally heterogeneous model [8]. This model predicts that (i) the $\mathrm{DO}_{3}$ nanoprecipitates undergo a cubic $\rightarrow$ tetragonal displacive transformation on cooling from nanoprecipitates that have a bcc-based $\mathrm{DO}_{3}$ structure to ones with an fctbased $\mathrm{DO}_{22}$ structure and (ii) the $c / a$ ratio of the $\mathrm{DO}_{3}$-like nanoprecipitates is large, on the order of 1.15. Our data indicate that the tetragonal distortion is less than that predicted for the $\mathrm{DO}_{3} \rightarrow L 1_{2}$ Bain strain by a factor of $\sim 3$, possibly because the transformation is incomplete and elastically constrained by the $A 2$ matrix. The large transformational strain raises the elastic energy too much in the $A 2$ matrix, preventing the growth of large $\mathrm{DO}_{22}$ precipitates and the expression of its complete distortion.

In summary, neutron diffuse scattering measurements have revealed (i) the importance of static-displacement modulations that accompany ordered $\mathrm{Fe}-\mathrm{Ga}$ alloys, although the results cannot be explained solely on the basis of a size effect in a conventional homogenous solution, and (ii) the presence of a local structural distortion with a peak splitting of $c / a \approx 1.04$. These findings show that the crystal structure of $\mathrm{DO}_{3}$ nanoprecipitates within the $A 2$ matrix is, in fact, strongly distorted from cubic symmetry, having a local symmetry of tetragonal or lower. Investigations as a function of Ga content showed that the diffuse scattering intensity is strongest near $\mathrm{Fe}_{0.81} \mathrm{Ga}_{0.19}$, where the magnetostriction is maximal: This confirms that the enhanced magnetostriction is directly related to the structural heterogeneity of tetragonally distorted $\mathrm{DO}_{3}$-like nanoprecipitates. Since the submission of our manuscript, an interesting paper has been published on the direct measurement of intrinsic atomic scale magnetostriction [15]. These studies were performed using a complementary method of differential x-ray absorption spectroscopy and clearly show the importance of local structure on magnetostriction.

This research was sponsored by the Office of Naval Research under Grants No. MURI N00014-06-1-0530 and No. N00014-06-1-0204. The authors are grateful to Armen Khachaturyan and Sy Moss for insightful discussions.

[1] A. E. Clark, J. B. Restorff, M. Wun-Fogle, T. A. Lograsso, and D. L. Schlagel, IEEE Trans. Magn. 36, 3238 (2000).

[2] A. E. Clark, M. Wun-Fogle, J. B. Restorff, T. A. Lograsso, A. R. Ross, and D. Schlagel, in Proceedings of the 7th International Conference on New Actuators, edited by $\mathrm{H}$. Borgmann (Messe Bremen GmbH, Bremen, Germany, 2000), p. 1111.

[3] O. Ikeda, R. Kainuma, I. Ohnuma, K. Fukamichi, and K. Ishida, J. Alloys Compd. 347, 198 (2002).

[4] H. Okamoto, in Phase Diagrams of Binary Iron Alloys, edited by H. Okamoto (American Society for Metals, Metals Park, OH, 1993), p. 147.

[5] A. E. Clark, M. Wun-Gogle, J. R. Restorff, T. A. Lograsso, and J. R. Cullen, IEEE Trans. Magn. 37, 2678 (2001).

[6] A. E. Clark et al., J. Appl. Phys. 93, 8621 (2003).

[7] M. Wuttig, L. Dai, and J. Cullen, Appl. Phys. Lett. 80, 1135 (2002).

[8] A. G. Khachaturyan and D. Viehland, Metall. Mater. Trans. A 38A, 2308 (2007).

[9] S. Bhattacharyya, J. R. Jinschek, A. Khachaturyan, H. Cao, J. Li, and D. Viehland, Phys. Rev. B 77, 104107 (2008).

[10] A.E. Clark, M. Wun-Fogle, J.B. Restorff, and T. Lograsso, in Proceedings of the Fourth Pacific Rim International Conference on Advanced Materials and Processing (PRICM4), edited by S. Hanada, Z. Zhong, S. Nam, and R. Wright, (The Japan Institute of Metals, Sendai, 2001), p. 1711.

[11] Hu Cao, Feiming Bai, Jiefang Li, D. Viehland, Thomas A. Lograsso, and Peter M. Gehring, J. Alloys Compd. 465, 244 (2008).

[12] Xuegen Zhao, Nigel Mellors, Susan Kilcoyne, Don Lord, Nicoleta Lupu, Horia Chiriac, and Paul F. Henry, J. Appl. Phys. 103, 07B320 (2008).

[13] B. E. Warren, B. L. Averbach, and B. W. Roberts, J. Appl. Phys. 22, 1493 (1951)

[14] Victoria M. Nield and David A. Keen, Diffuse Neutron Scattering from Crystalline Materials (Oxford University Press, New York, 2001), pp. 49-50.

[15] M. Ruffoni et al., Phys. Rev. Lett. 101, 147202 (2008). 